\title{
Metodología de diseño de una escotilla de inspección para recipientes a presión
}

\section{Inspection hatch for pressure vessels design methodology}

\author{
Andrés Mauricio Moreno Uribe \\ Estudiante Maestría en Ingeniería \\ Mecánica, Universidad Federal de \\ Minas Gerais, Brasil. \\ Correo electrónico: \\ andresmauriciomu@ufmg.br
}

Ezequiel C.P. Pessoa

PhD Ingeniería Mecánica -

Docente, Universidad Federal de

Minas Gerais, Brasil.

\author{
Alexandre Queiroz Bracarense \\ PhD. Ingeniería Metalúrgica y de Materiales - \\ Docente, Universidad Federal de Minas \\ Gerais, Brasil.
}

Gabriel Mendes de Almeida Carvalho Estudiante Maestría en Ingeniería Mecánica, Universidad Federal de Minas Gerais, Brasil.

Nilo Nogueira da Silva

Estudiante Doctorado en Ingeniería Mecánica, Universidad Federal de Minas Gerais, Brasil.

Información del artículo: recibido: junio de 2016, aceptado: septiembre de 2016 https://doi.org/10.17081/invinno.5.1.2613 


\section{Resumen}

$\mathrm{E}$ prescion luación de tensiones equivalentes en recipientes a presión mediante el método de elementos finitos. Con base en las consideraciones resultantes, se diseñó una escotilla de inspeccióny se realizaron comprobaciones analíticas por medio de técnicas de simulación por computador. Esto generó puntos de comparacióny un estudio sistemático con el fin de comprobar el comportamiento de las fuerzas resultantes en función de lasmodificaciones geométricas. También se definió un modelo a partir de tres propuestas de Manhole. Las configuraciones de escotilla de inspección responden a los formatos circular, elíptico-longitudinal y elíptico-transversal. Así, fue comprobado el trazado de tensiones sobre el objeto de estudio y se definió un procedimiento más incluyente en comparación con el método convencional, el cual se basa en expresiones de cálculo simplificadas.
Palabras clave:

Recipiente a presión, diseño, método de elementos finitos, escotilla de inspección, diseño asistido por computador, ingeniería asistida por computador.

\begin{abstract}
$\mathrm{T}$ his paper aims to describe the design methodology used for equiva- lent stresses assessment in pressure vessels through the application of the finite element method and based on the resulting considerations,

the creation of an inspection hatch design.Computer simulation technique and analytical checks were carried out. As the result, points of comparison were generated and a systematic study to test the behavior of the resulting forces based on geometric modifications were developed. A model along with three proposals of Manhole was defined. Inspection hatch configuration respondsto circular, elliptic-longitudinal and elliptictransversal for- mat. Thus, the layout of tensions about the subject of study was confirmed and also, a more inclusive procedure compared with conventional method, which is based on simplified calculation expressionswas defined.
\end{abstract}

Keywords:

container, design, finite element method, inspection hatch, computer aided engineering, computer-aided design. 


\section{INTRODUCCIÓN}

En oposición a lo que sucede con la mayoría de los elementos y componentes de máquinas y equipos industriales, los recipientes a presión son construidos sobre medida y por encomienda, atendiendo a finalidades o condiciones de desempeño determinadas [1]. A fin de estandarizar, hay normas internacionales que definen factores de seguridad para las partes que constituyen estos equipamientos. Sin embargo, de manera general, las normas de diseño establecen fórmulas simples asociadas a grandes coeficientes de seguridad, para compensar las abstracciones y simplificaciones. Esto ocasiona grandes errores de cálculo y el aumento de los costos del proyecto. Además, estos cálculos analíticos no permiten visualizar el comportamiento del modelo (recipiente a presión) bajo la acción de diferentes cargas combinadas, geometrías complejas y definición de condiciones de frontera.

Con la creciente adopción de métodos de resolución de ecuaciones diferenciales a dominios de cálculo complejos en todas las áreas de la ciencia, esta alternativa de análisis resulta ser un método fácilmente adaptable a casos prácticos.

\section{METODOLOGÍA}

\section{Geometría del modelo}

El primer paso en el desarrollo de este estudio fue la generación del modelo a partir de los planos originales del proyecto y su corroboración en campo, mediante la medición de las principales dimensiones del recipiente (Tabla 1). Así, con el uso del software SolidWorks (Education edition), se generó el cuerpo con un formato de pieza única, con la intención de simplificar relaciones de contacto entre componentes (en el caso de ensamblajes) y aligerar las condiciones de discretización. El "medio continuo" analizado tiene una forma cilíndrica (casco) y una posición de instalación horizontal; además, está constituido por dos tapas toriesfericas y una abertura que finaliza con un cierre entre una brida de cara plana y una brida ciega.

\section{Tabla 1. Principales dimensiones del recipiente a presión}

\begin{tabular}{|c|c|c|c|}
\hline Componente & D escripción & $\begin{array}{c}\text { Espesor } \\
(\mathrm{mm}) \\
{[\mathrm{in}]}\end{array}$ & $\begin{array}{c}\text { Diámetro } \\
\text { interno } \\
(\mathrm{mm})[\mathrm{in}]\end{array}$ \\
\hline $\begin{array}{c}\text { Casco o } \\
\text { membrana }\end{array}$ & $\begin{array}{c}\text { Formato de } \\
\text { Cilindro }\end{array}$ & $\begin{array}{c}6,35 \\
{[1 / 4]}\end{array}$ & $762[30]$ \\
\hline Tapa & $\begin{array}{c}\text { Tipo } \\
\text { toriesferica }\end{array}$ & $\begin{array}{c}7,9375 \\
{[5 / 16]}\end{array}$ & $762[30]$ \\
\hline Brida plana & $\begin{array}{c}\text { Cierre con } \\
\text { brida ciega }\end{array}$ & 60 \\
{$[2,3622]$} & 393,70 \\
{$[15]$}
\end{tabular}

El recipiente es soportado por dos bases o silletas de construcción metálica (Figura 1), las cuales, configuradas asimétricamente en relación a la longitud de tangencia entre las tapas, tienen como característica principal un ángulo de contacto con la circunferencia del tanque y nervaduras que actúan como refuerzos. Asimismo, el empalme casco-tapa toriesferica y casco-cuello de abertura representa regiones de transición de espesura y de formato, respectivamente, donde la desigualdad de la resistencia entre las partes, resulta en discontinuidades que introducen altas tensiones localizadas [1]. 


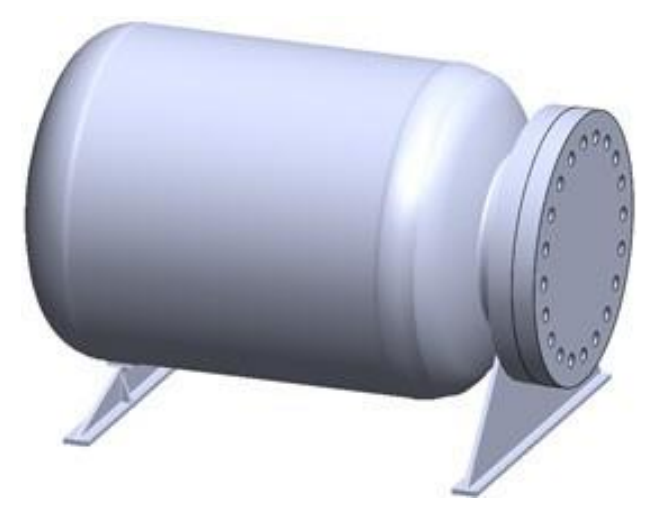

Figura 1. Perspectiva dimétrica del recipiente a presión

\section{Definición del análisis}

Luego de evaluar consideraciones geométricas, se definió el tipo de análisis a realizar con base en la aplicación del código general para el diseño de calderas y recipientes a presión [2]. Estas normas se refieren en su mayoría a esfuerzos estáticos, por lo cual, las tensiones admisibles para la selección del material, están en función de la presión de trabajo y la temperatura. Este último aspecto es definido como la temperatura en la membrana y en general es la misma temperatura del líquido contenido en el recipiente a presión [3]. Para este caso particular, la temperatura no fue considerada, ya que el recipiente no se encuentra bajo el efecto de gradientes térmicos de gran connotación, como en intercambiadores de calor, donde la dilatación térmica del material y su resistencia mecánica limitan las tensiones admisibles en función del incremento de la temperatura. Por consiguiente, se define un análisis estático lineal, y se tiene en cuenta que la relación entre la resistencia y las cargas debe cumplir con requerimientos de confiabilidad y seguridad, mediante la evaluación del tipo de material, geometría y restricciones espaciales. De este modo, la primera suposición realizada en este tipo de estudio (suposición estática), establece que la fuerza o el par de torsión aplicados a un elemento no cambian de magnitud, dirección o punto de aplicación [4], es decir, las cargas internas o externas, etc., son cargas estáticas. Estas se aplican lentamente sobre el modelo hasta llegar al máximo valor establecido, despreciando fuerzas inerciales y de amortiguamiento [5].

Además, para este tipo de análisis, debe considerarse la suposición lineal ; de lo que lógicamente se desprende que la relación entre cargas y respuestas inducidas también sea lineal, y que el material del modelo responde a la Ley de Hooke. En este caso también son consideradas la isotropía y homogeneidad. La primera establece que el cuerpo mantiene los mismos valores en términos de propiedades mecánicas en todas las direcciones, y la homogeneidad especifica que el material no posee ninguna discontinuidad o falla interna [6].

\section{Datos de entrada}

La definición del material que constituye el modelo se realiza mediante la selección de Acero Estructural en la biblioteca de recursos del software ANSYS Workbench, la cual suministra materiales para análisis de sistemas. Sus principales propiedades y sus respectivos valores son listados en la Tabla 2. El recipiente de este caso de estudio trabaja con una presión interna de operación de 1,5 MPa, simulando una profundidad aproximada de $150 \mathrm{~m}$ bajo el nivel del mar. Asimismo son consideradas fuerzas que actúan sobre el sólido, como la gravedad estándar de la tierra y el peso de la masa de agua distribuida sobre todas las caras internas del recipiente. 
En relación con las restricciones, el modelo fue posicionado y anclado en el entorno por medio de soportes fijos en la cara inferior de las silletas.

Tabla 2. Propiedades del Acero Estructural

\begin{tabular}{|c|c|c|}
\hline Propiedad & Valor & Unidad \\
\hline $\begin{array}{c}\text { Coeficiente } \\
\text { de } \\
\text { Expansión } \\
\text { térmica }\end{array}$ & $1,20 \mathrm{E}-05 \mathrm{~K}$ & \multirow{-}{*}{1} \\
\hline $\begin{array}{c}\text { Módulo de } \\
\text { Young }\end{array}$ & $2 \mathrm{E}+05$ & $\mathrm{MPa}$ \\
\hline $\begin{array}{c}\text { Coeficiente } \\
\text { de Poisson }\end{array}$ & 0,3 & - \\
\hline $\begin{array}{c}\text { Módulo } \\
\text { cortante }\end{array}$ & 76923 & $\mathrm{MPa}$ \\
\hline $\begin{array}{c}\text { Límite } \\
\text { elástico }\end{array}$ & 250 & $\mathrm{MPa}$ \\
\hline $\begin{array}{c}\text { Límite de } \\
\text { tracción }\end{array}$ & 460 & $\mathrm{MPa}$ \\
\hline Densidad & 7850 & $\mathrm{Kg} / \mathrm{m} 3$
\end{tabular}

Fuente: Adaptado del Engineering Data de ANSYS Workbench.

Calibración del modelo

Como método de "calibración" del análisis por elementos finitos, se realizó la comparación entre las tensiones resultantes del uso de fórmulas analíticas de acuerdo con el código ASME y el análisis de tensiones generado por el CAE.

Teniendo en cuenta que uno de los componentes principales de los recipientes a presión es esencialmente un sólido a revolución (cilindro), se evaluaron las tensiones que actúan sobre el casco para determinada presión y sobre un punto de vista simplificado del balance de fuerzas. Así, es bien conocido que en los cuerpos cilíndricos, las tensiones adctúan en las direccंmenes longitudinal ( ) y circunferencial ( ) [7]. La norma que rige el diseño de recipientes a presión [2] establece esas tensiones como sigue en (1) y (2):
Tensión circunferencial:

S_C $=(\operatorname{Pr} / t)+0,6 \mathrm{P}$

Tensión longitudinal:

S_L $=(\operatorname{Pr} / 2 \mathrm{t})-0,4 \mathrm{P}$

De las expresiones anteriores, “ $\mathrm{P}$ " , " $\mathrm{r}$ " $\mathrm{y}$ " $\mathrm{t}$ " son respectivamente presión $(\mathrm{Pa})$, radio interno (m) y espesura del casco (m). Reemplazando los datos de la Tabla 1, se obtuvo que la tensión circunferencial y longitudinal tienen valores de 90,9 MPa y 44,4 MPa respectivamente.

Luego se diseñó la membrana con las dimensiones usadas en los cálculos anteriores y se simuló el modelo mediante un análisis estático lineal. La Figura 2 presenta el análisis de tensiones en el casco, denotando un valor máximo y mínimo.

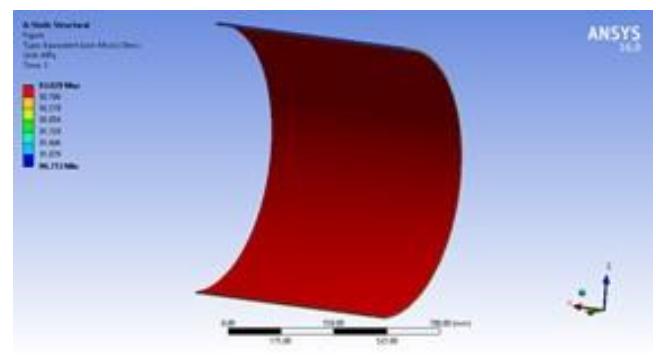

Figura 2. Sección del casco sobre presión

Como puede notarse, la paleta de colores al lado izquierdo de la figura, enmarca el rango de valores en los que se encuentra la tensión circunferencial en el elemento, la cual representa la tensión gobernante y de mayor importancia al momento de calcular. Así, fue validado el uso del software de elementos finitos para análisis de tensiones. La mínima divergencia entre los resultados analíticos y computacionales es consecuencia de las diferencias que conlleva el uso de los dos métodos, 
las cuales incluyen generación de mallas, refinamientos de las mismas, etc.

Es importante resaltar en este punto que el análisis de tensiones con el uso de un CAE se realizó según el criterio de falla conocido como "Teoría de la energía de distorsión para materiales dúctiles" (o "Tensión de Von Mises" como es mayormente difundido), que, en términos generales, permite conocer si la tensión está dentro de la zona elástica y óptima o alcanzó la zona plástica [5]. Aunque este criterio sea más incluyente por su forma característica de elipse en la representación de los estados de esfuerzo plano, el criterio de Tresca es considerado más conservador, por lo cual fue el adoptado en la continuación de la norma para diseño de recipientes a presión [8]. Sin embargo, para el caso de estudio en cuestión, la división 1 abarca la presión de operación impuesta al recipiente y exige menores controles de inspección.

\section{Evaluación de las tensiones y factor de seguridad}

El recipiente de presión evaluado, es el modelo simplificado del tanque para simulación de soldadura subacuática del Laboratorio de Robótica, Soldadura y Simulación de la UFMG. Con el objetivo de aplicar nuevos procedimientos de investigación, fue necesario realizar cambios en el equipamiento para cumplir con los estándares. Por esta razón, se realizó una verificación de tensiones para conocer el comportamiento aproximado del recipiente en las cargas impuestas y cómo será la respuesta a modificaciones geométricas que, en este estudio, involucran una escotilla de inspección y, por ende, una nueva abertura.

Se sabe que "la retirada de una sección parcial de pared de un casco sometido a presión interna, para lainstalación de una boquilla, genera una concentración de fuerzas en los alrededores de la abertura" [7]. Lo anterior estipula nuevas consideraciones en el proceso de diseño, que deben compensar el efecto de la fragilidad del elemento causada por la brecha. La Figura 3 presenta un esquema de la concentración de fuerzas en aberturas.



Figura 3. Concentración de fuerzas en aberturas. $\mathrm{P}$ : presión, d: diámetro de abertura, $r$,t: radio y espesor del casco, F: fuerzas en la cercanías de la abertura, $f$ : fuerzas en áreas de membrana. Adaptado de [7]

Cabe considerar, que el resultado de las fórmulas simples usadas en la calibración del modelo (sección anterior), sólo ofrece una relativa precisión en las llamadas "áreas de membrana", que son regiones de la pared del recipiente alejadas de cualquier mudanza de formato y de cualquier accesorio (aberturas, tapas de registro, etc.). Por ello, para otras secciones del recipiente, el margen de error resultante de esos cálculos simples puede ser grande [1]. Casi todas las normas de diseño establecen estas fórmulas simples asociadas a grandes coeficientes de seguridad para varios elementos de un recipiente a presión a fin de compensar las abstracciones y simplificaciones hechas en estas expresiones. Sin embargo, aunque el cálculo matemático para análisis de tensiones y el uso de ecuaciones genere valores para 
evaluar el diseño, estos métodos (que básicamente son los adoptados por las normas actuales) no consideran mudanzas de formato como tampoco el comportamiento de las tensiones en algunas partes del recipiente ni combinaciones de cargas, por lo que no analizan el recipiente a presión como un todo, sino como un conjunto de elementos.

Visto de esta forma, la filosofía general de la norma de diseño aplicada en esta metodología consiste básicamente en limitar las tensiones en los componentes del recipiente a presión (casco, tapas y aberturas) a una fracción de una característica mecánica de los materiales (límite elástico), para luego, con los métodos matemáticos aplicados con el uso de un software CAE, evaluar con precisión las tensiones que gobiernan cada punto del modelo.

A continuación, se define el trazado de factor de seguridad (SF) sobre el modelo. Este responde al criterio de máxima tensión de Von Mises-Hencky y expone esencialmente que un material dúctil comienza a ceder en una ubicación cuando la tensión de Von Mises es igual o superior al límite de tensión específico (límite elástico). De este modo, la seguridad del diseño responde a la expresión enmarcada en (3):

F a c tor d e S e gurid a d $\frac{\sigma \text { limite elástico }}{V \text { onm ises }}$

Con todo, en este estudio se consideró que un SF $\leq 1$ representa fallas de material en la ubicación; en tanto que para valores mayores a la unidad, el material no representa riesgos en un determinado punto.

Formato de la Abertura de escotilla y tapa de acceso rápido

Según [6], las boquillas de visita (escotillas de inspección) son aberturas que permiten la entrada de personas al interior del recipiente para limpieza, mantenimiento, montaje y desmontaje de piezas, y son bloqueadas por medio de tapas removibles. El código ASME (ASME section VIII Division 1, 2013) permite Manholes circulares, elípticas u ovales con dimensiones mínimas de $380 \mathrm{~mm}, 280 \times 380 \mathrm{~mm}$ y $250 \times 400$ $\mathrm{mm}$, respectivamente.

Ahora bien, los tipos de Manhole evaluados en el presente estudio fueron los de formato circular y elíptico. El pasaje elíptico garantiza una mayor comodidad para el operador que ingresa en el recipiente [9]. Este formato fue analizado en dos configuraciones: el formato elíptico-transversal, posicionado en forma perpendicular al eje longitudinal del cilindro y el formato elíptico-longitudinal, en el cual el semieje que describe el diámetro mayor de la elipse está paralelo al eje longitudinal del cilindro.

Al mismo tiempo, las tapas pueden tener varios formatos. Los evaluados en esta metodología son los siguientes:

- Tapa plana: son usadas para recipientes a presión atmosférica, teniendo como principal característica, un bajo costo de fabricación [10].

- Tapa Toriesferica: Tiene bajo costo de fabricación y soporta considerables presiones manométricas.

- Tapa Elíptica: Su sección trasversal es una elipse geométrica perfec

generalmente cumplen con una relación 2:1.

Adicionalmente, al trabajar con altas presiones internas, la escotilla de inspección debe abrir hacia el interior del tanque; debido a esto, se hace necesario que el Manhole penetre el espesor del recipiente hacia el interior de este (Figura 4), con el objetivo de garantizar un perfil plano sobre el cual realizar el asiento del borde de la tapa. 


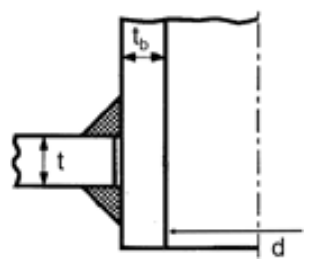

Figura 4. Configuración representativa describiendo la entrada de la boquilla en la membrana del recipiente a presión; t: espesor del casco, tb= espesor de boquilla, $d$ : diámetro de boquilla. Adaptado de [11]

\section{RESULTADOS Y DISCUSIÓN \\ Verificación de tensiones}

Con el objetivo de corroborar el estado del recipiente a presión para el diseño de la escotilla de inspección, fue simulada una sección de la membrana que representa 1/8 del recipiente a presión. Así se verificaron las tensiones equivalentes con las modificaciones sucesivas de la geometría. Esta sección incluye tapa y membrana. El trazado de tensiones y las deformaciones son presentados en las Figuras 5 y 6 .

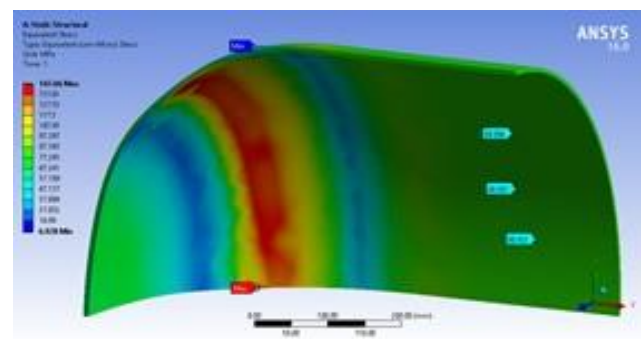

Figura 5 . Tensiones equivalentes en $\mathbf{1 / 8}$ del modelo

En la Figura 5, se observa que las tensiones equivalentes en el área de membrana adoptan un valor más realista en comparación con el que se obtuvo en la sección "Calibración del modelo". Lo anterior constituye la respuesta de una geometría más acorde (con la inclusión de la tapa) y un método de cálculo mejorado. También se detalla un aumento de las tensiones en la zona de transición entre la sección toroidal (knuckle) y la sección elíptica (cúpula central de la tapa). Este es un resultado, como consecuencia de la mudanza de formato. Cabe resaltar que este tipo de tapas (toriesfericas) son las más comunes y de mayor uso en la industria gracias a su fácil fabricación. El SF mínimo es de 1,7.

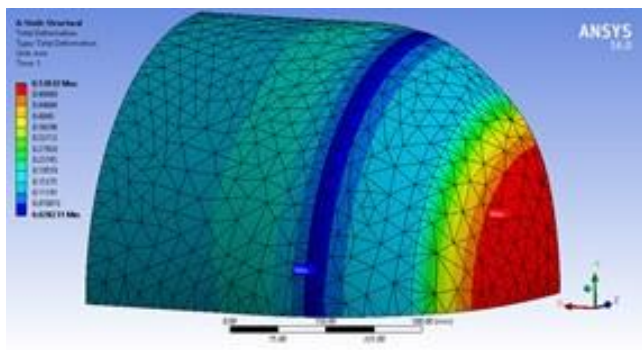

Figura 6. Deformación total sobre la sección del recipiente y mallado

Por outra parte, el gráfico de deformaciones de $1 / 8$ de sección de modelo (Figura 6 ) expone la discretización en los diferentes elementos de malla; para los propósitos simplificados de este análisis, la malla Por outra parte, el gráfico de deformaciones de 1/8 de sección de modelo (Figura 6) expone la discretización en los diferentes elementos de malla; para los propósitos simplificados de este análisis, la malla está compuesta de 6626 elementos y 5572 nodos. Además, las mayores deformaciones se encuentran en la cúpula de la tapa, con un máximo de $0,53 \mathrm{~mm}$.

Sobre la base anterior, se modificó la geometría del modelo, instalando la abertura principal y la brida de cierre. Para esta simulación se usó el software SolidWorks Simulation (Education Edition), el cual se integró al CAD SolidWorks®. Las Figuras 7 y 8 relacionan las tensiones equivalentes $y$ el trazado de factor de seguridad (SF), respectivamente. 


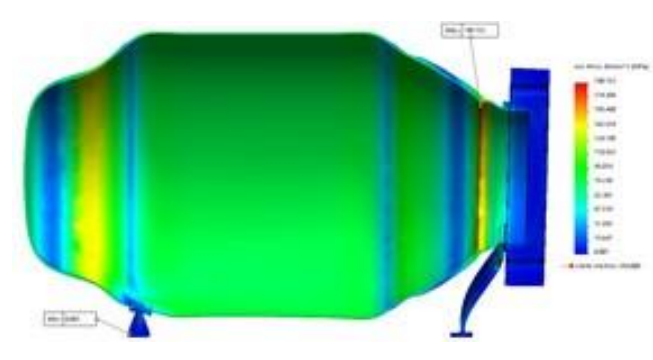

Figura 7. Tensión de Von Mises para modelo con abertura



Figura 8. Trazado del Factor de seguridad (SF)

Como se esperaba, las máximas tensiones se hallan en la abertura, que son definidas en la literatura como tensiones secundarias [1]: básicamente son el resultado de restricciones geométricas en el propio recipiente. De este modo, en este caso específico, la transición de formato es el motivo del cúmulo de tensiones de flexión causadas por fuerzas y momentos que actúan en la abertura [7]. En lo que se refiere al trazado de $\mathrm{SF}$, el software proporciona un valor mínimo de 1,315 en el cuello de la abertura. El esquema de la parte superior izquierda de la Figura 8, que representa un trazado "iso" de tensiones em el que los volúmenes en rojo simbolizan la ubicación de material con un SF menor igual a 1,6. Algunas normas desconsideran este tipo de tensiones por tener una naturaleza auto limitante, ya que son aliviadas como resultado de pequeñas deformaciones

plásticas, alcanzando como tensión máxima la propia tensión elástica del material, pero sin resultar en mayores deformaciones.

En lo que respecta a la imagen del rectángulo de borde oscuro, se introduce un ejemplo de tensiones localizadas máximas o de "pico". La unión entre la silleta y el soporte triangular causa una concentración de tensiones como resultado de la discontinuidad geométrica del modelo en ese punto. Sin embargo, estas tensiones no representan riesgos a la integridad del recipiente, pues actúan en una región limitada. Con este software, la malla sólida es compuesta de elementos sólidos tetraédricos (con 4 puntos jacobianos), una calidad de malla para elementos cuadráticos de alto orden, con un total de 43954 elementos y 81982 nodos.

\section{Evaluación del tipo de abertura (Manhole)}

En función del requerimiento de las nuevas técnicas de investigación para el recipiente a presión, se planteó una abertura sobre la parte superior de la membrana, fijando una distancia de $200 \mathrm{~mm}$ desde la línea de tangencia entre casco y tapa toriesferica, hasta el centro de la abertura. Como muestra la Figura 9, se considera sólo una sección de la membrana luego de la abertura, ya que, como es sabido, después de cierta longitud, las tensiones alcanzan un valor aproximado al valor de la tensión equivalente en el área de membrana [6]. En la misma figura son presentados los esquemas de diseño de los tres formatos de Manhole y algunas dimensiones: 


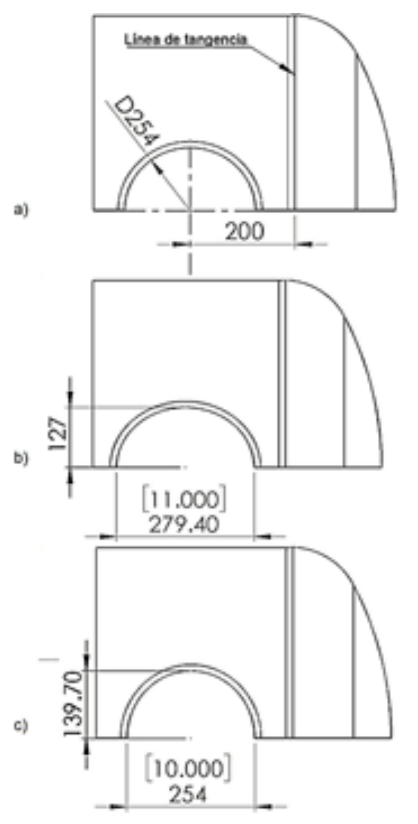

Figura 9. Abertura de Manhole en formato: a) circular, b) elíptico-longitudinal, c) elíptico-transversal

La abertura libre para el formato circular tiene un diámetro de $254 \mathrm{~mm}$ [10 in], yel formato elíptico de $254 \mathrm{~mm}$ [10 in] y 279,4 $\mathrm{mm}$ [11 in]. La boquilla fue diseñada del mismo material que la membrana y tiene $58 \mathrm{~mm}$ de altura desde la generatriz superior del recipiente, y un espesor de 12,7 mm [0,5 in]. También se aplicaron controles de refinamiento de malla en las aristas de los tres formatos, ya que por recomendación [9], son los puntos de estudio gobernantes (junta de abertura/membrana), como muestra la Figura 10.

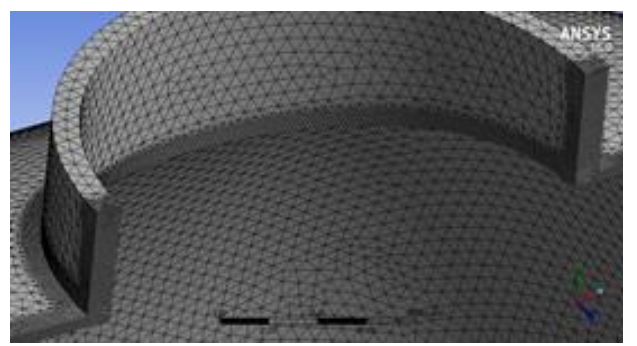

Figura 10. Refinamiento de malla en Manhole

Para las tres variantes consideradas según los propósitos de este estudio, las mallas son similares, es decir, están compuestas por 106652 elementos y un total de 179407 nodos. Las cargas de presión fueron establecidas en función de la constante gravitacional de la tierra y las sujeciones. Como puede ser detallado en la Figura 11, las isolíneas representan por medio de áreas la distribución de tensiones equivalentes, donde se evidencian altos valores en la región de unión entre abertura/membrana. Lo anterior confirma la teoría de aumento de tensiones como consecuencia de aberturas en el casco de recipientes a presión. En cuanto al SF, el valor mínimo es de 0,9586, definiendo una falla de material en la ubicación de la máxima concentración de tensiones.



Figura 11. Isolíneas de distribución de las tensiones equivalente para Manhole Elíptico-longitudinal

Posteriormente, fue establecido un estudio de las tensiones equivalentes sobre la geometría anterior (Von Mises) en relación con la longitud. Según [9]. Se esperaría que en el caso en que el Manhole se sitúe cerca de una transición de formato membrana/ tapa toriesférica, colisionen las tensiones que se producen por los tipos de discontinuidades. Así, fueron seleccionados dos puntos mostrados en la Figura 12, entre el final de la abertura para Manhole-circular y el inicio de la sección cilíndrica para unión entre membrana y tapa toriesferica. 
Como puede corroborarse en tal figura, la tensión equivalente disminuye a medida que se aleja de la abertura: desde una tensión de 219,76 MPa en el punto 1, hasta $54,651 \mathrm{MPa}$, para el punto 2, pasando por un máximo (representado con la banda de contorno roja de la parte superior del gráfico y el punto rojo en la curva).

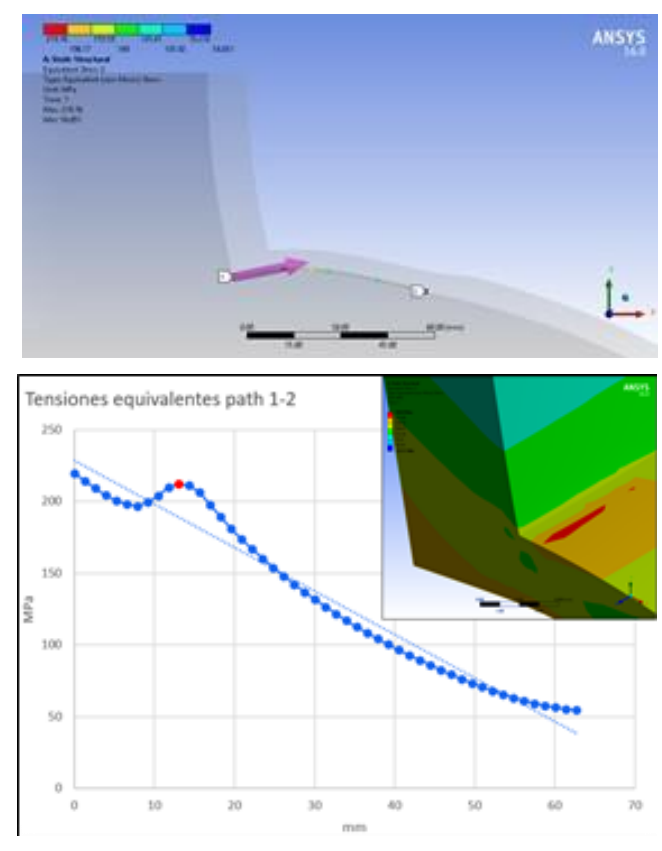

Figura 12. Recorrido de tensiones Equivalentes entre abertura y unión de membrana/tapa toriesférica para Manhole circular

Ahora bien, las deformaciones del modelo siguen presentándose con mayor intensidad en la tapa toriesferica (del orden de $0,55 \mathrm{~mm}$ ). De manera semejante, a un costado de la membrana y por debajo de la abertura del Manhole, se presenta un área de deformación con valores cercanos a la cúpula de la tapa (Figura 13).

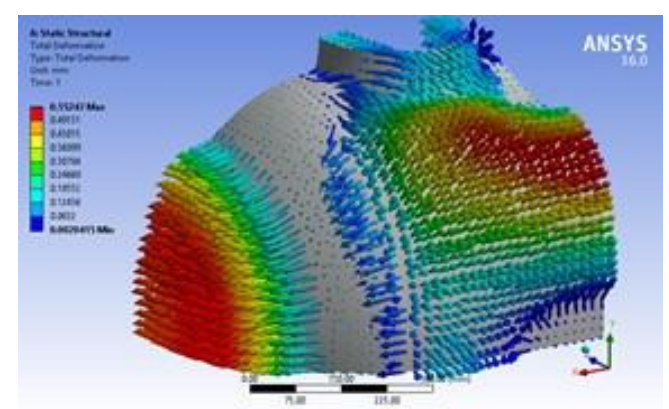

Figura 13. Deformación del recipiente a presión con diseño de Manhole circular

La escotilla de inspección con abertura elíptico-longitudinal se simuló bajo las mismas condiciones. La Figura 14 presenta superficies generadas a partir de isolíneas. En ella es posible comprobar la ubicación de las zonas con mayores tensiones, las cuales van desde la superficie interna de la membrana hasta la superficie exterior del recipiente. Como característica importante, la ubicación de las tensiones máximas (señalada por la marca oval de guiones) se trasladó hacia el lado más próximo a la mudanza de formato entre membrana/tapa toriesferica. Este fenómeno es descrito como resultado de una rigidez excesiva derivada de la unión entre el Manhole y la membrana [9].

La Figura 15 expone una estructura alámbrica deformada del modelo, en la cual, son ubicados los volúmenes con mayor deformación. A diferencia de los resultados encontrados en secciones anteriores, la zona de mayor deformación se encuentra en la membrana y es definida en 0,61 $\mathrm{mm}$. 


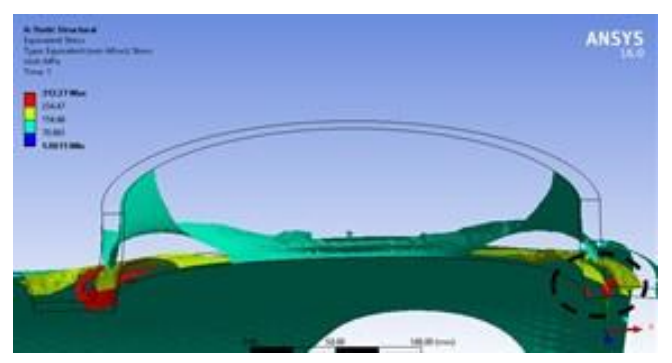

Figura 14. Superficies iso en Manhole elípti- colongitudinal

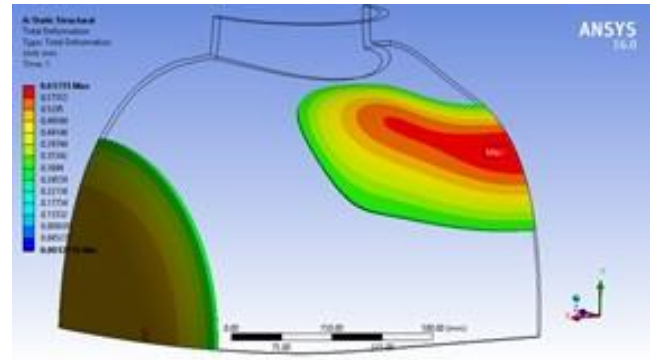

Figura 15. Volúmenes de mayor deformación en el modelo con escotilla de inspección elíptico-longitudinal

Por último, el formato elíptico-transversal presenta los mejores resultados (Figura 16), estableciendo una tensión máxima de 235,56 MPa, ubicada en la región más próxima del área de membrana. También las deformaciones disminuyen $(0,5396$ $\mathrm{mm}$ ) y se localizan en la cúpula de la tapa toriesferica. Esta configuración ofrece el factor de seguridad (SF) de valor mayor entre los tres formatos: 1,0613.

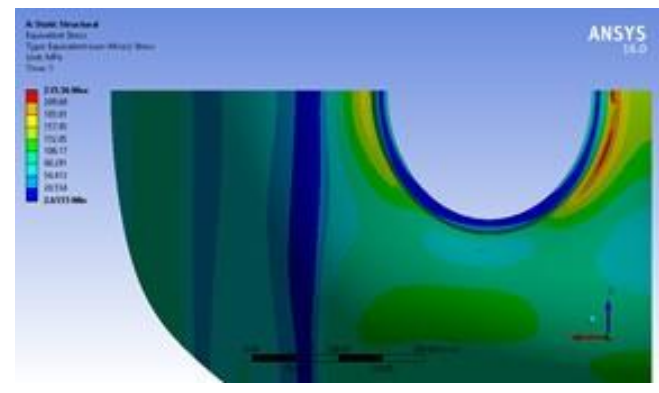

Figura 16. Distribución de tensiones equivalente para el formato elíptico-transversal
Ahora bien, representando la penetración de la boquilla del Manhole sobre la membrana del recipiente $(50 \mathrm{~mm}$ hacia el interior), en la Figura 17son presentadas las tensiones equivalentes de este procedimiento. Se observa que la tensión se encuentra entre 220,78 $\mathrm{MPa}$ y 0,10 $\mathrm{MPa}$, resultando en un menor rango de valores en comparación de la modificación para boquilla en formato elíptico-transversal sin penetración. De lo anterior, se puede inferir que la incrustación hacia el centro del recipiente por parte de la escotilla de inspección, representa una mejor distribución de las tensiones, generando menores valores. Las deformaciones siguen distribuyéndose como en simulaciones anteriores. Por obvias razones, el SF de esta simulación también incrementó, definiendo un valor mínimo de 1,13. Un resultado similar ya fue obtenido en la referencia [9]. Tal referencia indica (entre otras características) que la configuración de la escotilla de inspección en forma transversal genera un buen comportamiento y resultados prometedores.

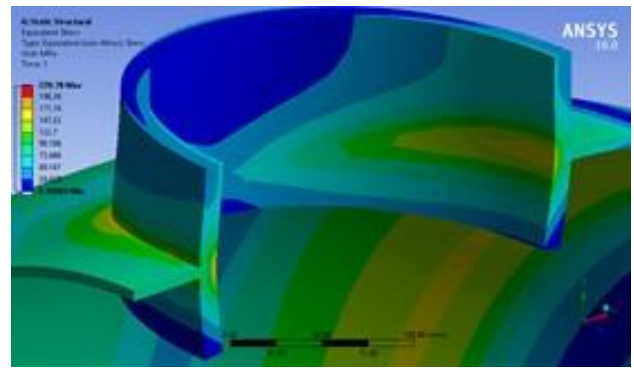

Figura 17. Tensiones equivalentes para Escotilla de inspección de formato elíptico-transversal con penetración en la membrana

También se realizó un trazado de tensiones sobre un path o ruta especificada, nuevamente entre dos puntos (1 y 2). Así, en la Figura 18, fueron graficadas las tensiones vs la longitud, que representan las 
tensiones en la región de unión entre la membrana y la abertura, alcanzando un máximo al inicio del formato elíptico del Manhole (marca de línea oscura al lado izquierdo) y apareciendo nuevamente sobre la línea oscura del gráfico en la parte derecha. Estas marcas limitan el inicio de la escotilla de inspección (la región entre marcas es la abertura libre, por lo tanto no se registran datos de tensiones).

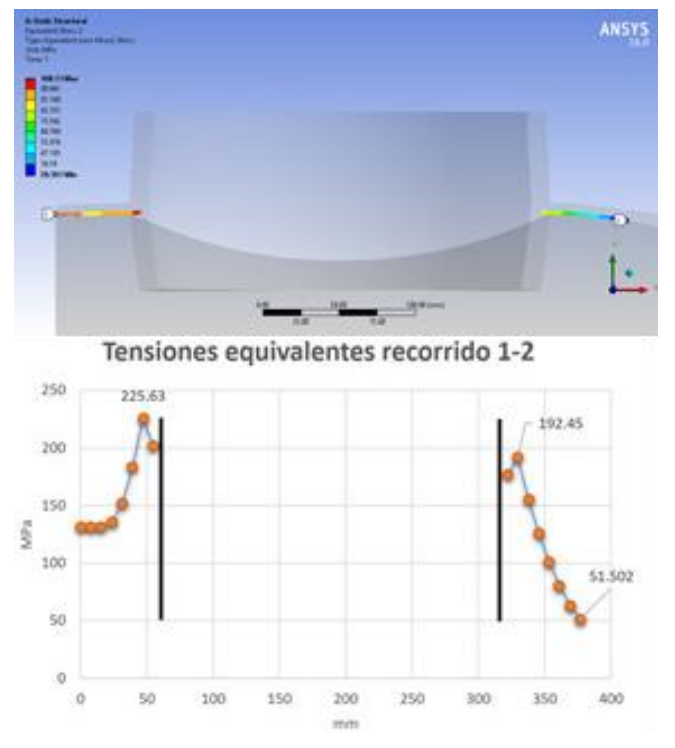

Figura 18. Recorrido de tensiones desde el área de membrana hasta el inicio del cambio de formato entre casco/tapa toriésferica

Por último, se evalúa el modelo modificado con el Manhole de formato elíptico-transversal, el cual presentó las menores tensiones equivalentes. La Figura 19 presenta la distribución de tensiones sobre $1 / 4$ del recipiente a presión. También, la Figura 20 expone las áreas de mayor concentración de tensiones desde una vista inferior. El SF mínimo para este análisis es de 1,058. Y, aunque el número que representa el factor de seguridad establezca que no se tienen fallas de material, este valor es poco conservativo. A modo de comparación, las tensiones de Von Mises del modelo final aumentan $24,24 \%$ en relación con las ten- siones equivalentes del modelo sin modificación para Manhole (Figura 7). De este modo, fueron visualizadas las respuestas en función de las cargas impuestas y las condiciones de contorno apropiadas para cada sección del modelo. Lo anterior establece un método basado en el FEM para el análisis estático de recipientes a presión.

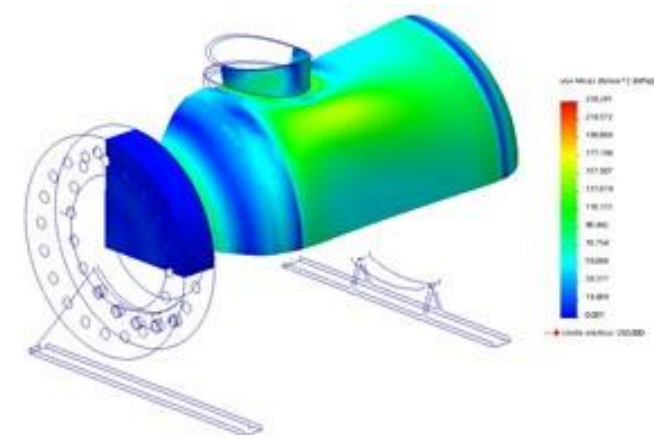

Figura 19. Visualización de las tensiones equivalentes sobre el modelo configurado con una escotilla de inspección de formato elíptico - transversal

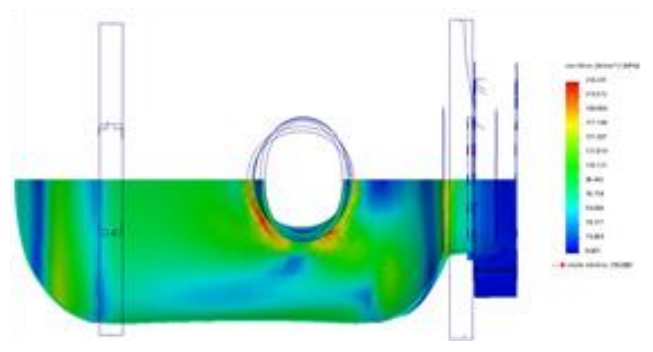

Figura 20. Vista inferior del modelo final

\section{Evaluación del tipo de Tapa}

Dando continuidad al estudio, se evaluaron 3 tipos de tapasdiseñadas en función del espesor de la boquilla del Manhole, con el objetivo de lograr el asiento entre ese conjunto. Este tipo de tapa no es especificado en las normas para diseño de recipientes a presión, debido a que estas no cuentan con la pestaña para efectuar el cierre. Por tanto, se incluyó la pestaña 
para el asiento, como se evidencia en la Figura 21.



Figura 21. Sección de Tapa Toriesferica con formato circular

Enseguida, se analizaron los 4 tipos de tapas(Figuras 22, 23, 24 y 25). En la evaluación fueron utilizados trazados de tensión equivalentes y factores de seguridad .

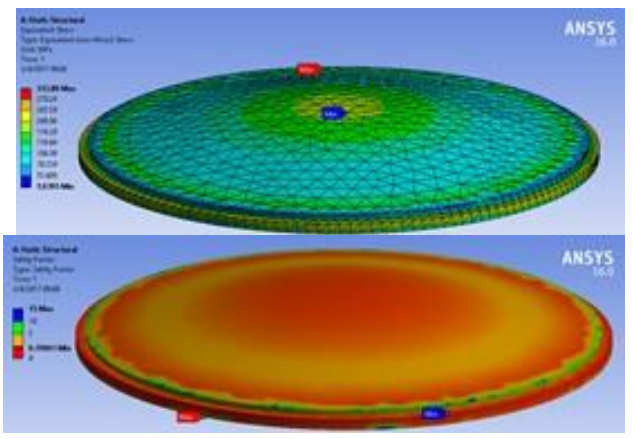

Figura 22. Trazado de tensiones equivalentes y factor de seguridad para tapa plana y Manhole circular

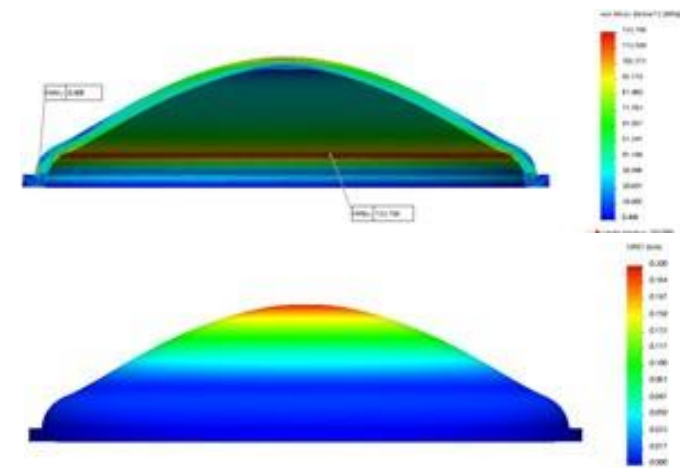

Figura 23. Trazado de tensiones equivalentes y factor de seguridad para tapa Toriesferica y Manhole circular

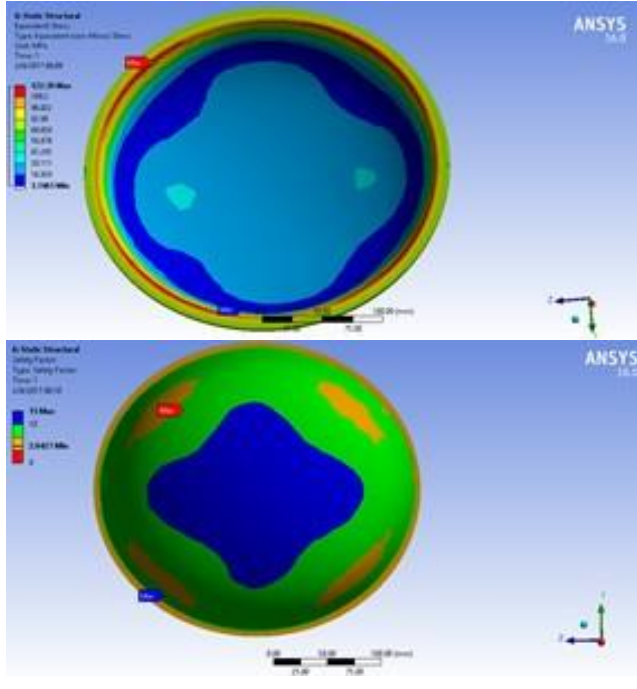

Figura 24. Trazado de tensiones equivalentes y factor de seguridad para tapa elíptica y Manhole elíptico



Figura 25. Trazado de tensiones equivalentes y factor de seguridad para tapa elíptica y Manhole circular

Las tensiones equivalentes mudan en función de la geometría; definiendo desde la tapa que ofrece mayor resistencia (menores tensiones) hasta la que tiene un SF menor; con esto se adoptó la siguiente nomenclatura: la Figura 22 fue designada con la letra A, la Figura 23 con la letra B, la Figura 24 con la letra $C$ y la Figura 25 con la letra D:

$$
\mathrm{D}>\mathrm{C}>\mathrm{B}>\mathrm{A}
$$

De este modo, la tapa elíptica para esco- 
tilla de inspección en formato circular presenta las tensiones más bajas para las cargas y sujeciones evaluados en la simulación, además de un alto factor de seguridad $(\mathrm{SF}=5,64)$.

\section{CONCLUSIONES}

La metodología aplicada en este estudio resulta ideal para las consideraciones que se evaluaron al delimitar el análisis. Tomando en cuenta que la modificación propuesta parte de un modelo existente, un análisis de tensiones sistemático y progresivo revela puntos de interés y permite descartar resultados críticos. De esta manera se pueden tomar decisiones de fondo y garantizar la convergencia de los resultados. Un ejemplo de esto es la ubicación de tensiones de pico, las cuales son relatadas en la literatura y que, gracias a la aplicación del FEM computacional, permiten ser visualizadas para verificar que no comprometen la integridad del recipiente a presión. Por otro lado, la facilidad de uso de una interfaz computacional y la completa integración entre el CAD y CAE posibilitan y simplifican las innúmeras modificaciones que se realizan, con el fin de obtener los mejores resultados.

Del estudio expuesto, se puede inferir de una manera más particular que:

- $\quad$ El método computacional para el análisis estático estructural se destaca de manera considerable sobre el método analítico, teniendo como principal característica la representación de tensiones equivalentes en el modelo como un todo y no componentes individuales.

- $\quad$ El análisis paulatino de tensiones sobre una geometría a modificar, permite conocer el estado del modelo y ofrece un punto de partida y comparación.

- El Manhole con formato elíptico-transversal presenta el mejor compor- tamiento respecto a las cargas y restricciones impuestas y en comparación con los formatos circular y elíptico longitudinal. - $\quad \mathrm{Al}$ contrario de los esperado por el autor de la referencia [9], la proximidad entre la abertura y la discontinuidad representada en la unión membrana/tapa toriesférica no produce una colisión de tensiones que comprometa la integridad del recipiente, dándose más bien un comportamiento de caída de la tensión en función de la longitud.

- La penetración del Manhole en la membrana del recipiente a presión representa una mejor distribución de tensiones y resulta en menores valores para el factor de seguridad.

- $\quad$ Como queda relatado por la literatura, a partir de la abertura, las tensiones equivalentes alcanzan valores semejantes a los que presentan las áreas de membrana.

- La tapa elíptica para el Manhole circular ofrece mayor resistencia en comparación con las otras configuraciones.

- Para un trabajo futuro, se recomienda una evaluación de la aplicación de suavizado (redondeo) en las aristas críticas de la unión membrana-Manhole.

- $\quad$ Por último, un análisis por fatiga permitiría reconocer puntos críticos y reconsiderar decisiones de diseño.

\section{AGRADECIMIENTOS}

Los autores agradecen al Laboratório de Robótica, Soldagem e Simulação (LRSS) de la Universidad Federal de Minas Gerais (UFMG), así como al Centro de Tecnologia e Mobilidade (CTM/UFMG) por permitir el uso del software SolidWorks (Educational Edition) y ANSYS. También se agradece por el apoyo financiero a la Coordenação de Aperfeiçoamento de Pessoal de Nível Superior (CAPES). 


\section{REFERENCIAS BLIBLIOGRÁFICAS}

[1] P. C. Da Silva Telles, Vasos de pressão, 2 edición, Rio de Janeiro, Brasil: LTC - Livros Técnicos e Científicos Editora S.A., 2001.

[2] ASME Boiler and Pressure Vessel Code, VIII Rules for Construction of Pressure Vessels Division 1, 2013.

[3] Pipe Flanges and Flanged Fittings, NPS 1/2 Through NPS 24 Metric/Inch Standard, 2013.

[4] Budynas y Nisbett, Shigley's Mechanical Engineering Desing, 8 edición, México: The McGraw-Hill Companies, Inc., 2008.

[5] S. Gómez González, SolidWorks Simulation, México: Alfaomega Grupo Editor, S.A. de C.V., 2010.

[6] G. Abreu Tomazini, "Diretrizes básicas para projetos de vasos de pressão segundo a ASME," Tesis trabajo de grado UNESP, Guaratinguetá, Brasil, 2015.

[7] C. Falcão, Projeto mecânico vasos de pressão e trocadores de calor casco e tubos, Revisão 2008, Brasil: Fundação Biblioteca Nacional do Ministério da Cultura, 2008.

[8] ASME Boiler and Pressure Vessel Code, VIII Rules for Construction of Pressure Vessels Division 2, 2013.

[9] J. Lewiński. "The Effect Of Manhole Shape And Wall Thickness On Stress State In A Cylindrical Pressure Vessel," En Journal Of Theoretical And Applied Mechanics, Vol.53, no. 1, pp. 59-68, 2015. [10] J. M. León Estrada, Diseño Y Calculo De Recipientes A Presión, Edición 2001, México: Ingeniería León S.A. de C.V., 2001.

[11] Design and Construction of Large Welded Low Pressure Storage Tanks, API 620, 2002.

\section{Este artículo se cita:}

A. Moreno, A. Queiroz, E. Pessoa, G. Men- dez, y N. Nogueira, "Metodologia de diseño de una escotilla de inspección para recipien- tes a presión, Revista Investigación e Innova- ción en Ingenierias, vol. 5, $\mathrm{n}^{\circ}$ 1. pp. 8 - 23, 2017. 